Z. Epileptol. 2019 · 32:177-182 https://doi.org/10.1007/s10309-019-0261-y Published online: 25 June 2019

(c) The Author(s) 2019

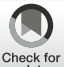

G. Kuchukhidze' $\cdot$ J. Höfler' $\cdot$ M. Kronbichler ${ }^{2,3} \cdot$ E. Schmid ${ }^{1}$ M. Kirschner' $\cdot$

L. Rainer' ${ }^{1}$ L. Kronbichler ${ }^{2,3} \cdot$ J. Gaggl' $\cdot$ E. Trinka ${ }^{1,3}$

'Department of Neurology, Christian Doppler Klinik, Paracelsus Medical University of Salzburg, Salzburg, Austria

${ }^{2}$ Neuroscience Institute, Christian Doppler Klinik, Paracelsus Medical University of Salzburg, Salzburg, Austria

${ }^{3}$ Centre for Cognitive Neuroscience, University of Salzburg, Salzburg, Austria

\title{
Emotion recognition and social cognition in juvenile myoclonic epilepsy
}

Neuropsychological studies revealed subtle cognitive deficits in patients with JME, mainlyimplicating the frontallobes, affecting attention and executive functions such as abstraction and categorization, planning, and mental flexibility [5, 6]. Brain network abnormalities associated with decision-making behaviors were shown mainly in JME patients with ongoing seizures compared with those with controlled seizures or healthy individuals $[6,7]$.

Psychiatric comorbidity is common in JME, affecting almost half of the patients [8]. In his seminal work, Dieter Janz described patients with JME as having rather unstable, suggestible, unreliable, and immature personalities [2]. Emerging evidence from the field of psychiatry suggests that patients with abnormal emotion processing and regulation, such as those with bipolar disorder, show disrupted connectivity between limbic structures and frontal cortices $[9,10]$.

There is an unmet need for studies on emotion and social-cognitive deficits and their morphological substrates in JME patients. Insights into pathophysiological mechanisms of emotion dysregulation and poor social adjustment would enable the development of new psychological and pharmacological interventional strategies for managing behavioral disturbances in patients with JME.

In this study, we aimed to address the problem of emotional disturbances and poor social adjustment in JME patients from multiple perspectives. We used thorough functional and structural assessments, which potentially enable elaboration of a unifying concept explaining the neurobiological background of disturbances in emotional processing and social adjustment in JME patients.

\section{Methods}

Patients aged 14 years or older with an electro-clinical diagnosis of JME were recruited. Healthy controls, including the siblings, were matched with patients with regard to age, sex, and education.

All patients underwent EEG and clinical assessment. Demographic data, age at seizure onset, duration of epilepsy, seizure types, seizure frequency, antiepileptic drug treatment, photosensitivity, psychiatric comorbidity, and previous psychological/psychiatric treatment were documented among other parameters.

The following tests were utilized in the study:

\section{SCID I and SCID II}

The Structured Clinical Interviews for DSM-IV Axis I (SCID-I) and Axis II (SCID-II) represent the American Psychiatric Association's official interview instruments for the assessment of DSMIV Axis I clinical disorders and Axis II personality disorders, respectively [11, 12]. elicited by cognitive activity [4] 
Facial Expressions of Emotion: Stimuli and Test

Facial Expressions of Emotion: Stimuli and Test (FEEST; [13]) contains validated series of photographs showing six basic emotions (happiness, fear, disgust, anger, sadness, surprise) in addition to neutral expressions.

\section{Empathy Quotient}

The Empathy Quotient (EQ; [14]) is a validated self-report questionnaire assessing the capacity to empathize with others, i.e., to recognize the affective state of another individual and to respond with an appropriate emotion.

\section{Moving Triangles}

The Moving Triangles test measures the extent to which subjects make mental state attributions to dynamic visual stimuli [15].

\section{Reading the Mind in the Eyes Test}

The Reading the Mind in the Eyes Test ("Eyes test"; [16]) measures higher-level facial emotion perception, where the subject has to infer mental and affective states from social cues of the eye region.

\section{Faux Pas Test}

The Faux Pas Test estimates the ability to recognize and understand a social faux pas, a statement in which the speaker accidentally offends or insults another person [17].

\section{Functional MRI}

Functional data were acquired using EPI $\mathrm{T} 2{ }^{*}$-weighted sequences with the following parameters: $3-\mathrm{mm}$ slice thickness (interslice gap: $1.2 \mathrm{~mm}$ ), FOV $250 \mathrm{~mm}$, matrix size $64 \times 64$ (voxel size $1.72 \times 1.72 \times 3.75 \mathrm{~mm}$ ), which allowed imaging of the complete temporal lobe, as well as frontal and parietal areas. Analysis of fMRI data was performed with the SPM12 (Statistical Parametric Mapping 12, Wellcome Trust Centre for Neuroimaging, Institute of Neurology,
UCL, London, UK). The data were realigned to account for motion, smoothed with a $5-\mathrm{mm}$ Gaussian smoothing kernel, and normalized to a standard template in Montreal Neurological Institute space.

\section{fMRI paradigms}

We assessed emotion recognition by a well-characterized "dynamic fearful faces" paradigm. For assessment of social cognition, a theory of mind (ToM) fMRI paradigm was employed.

\section{Statistical analysis}

The patients' coded data (clinical, imaging, psychiatry, neuropsychology) were analyzed with SPSS (IBM, Armonk, New York, USA). The patients' clinical characteristics were summarized using descriptive statistical methods, such as frequencies, percentage, means, and standard deviations. We performed a subgroup analysis by using descriptive statistics in an explorative way. Categorical data were analyzed by means of Pearson's chi-squared test (if all expected numbers of counts are at least 1); otherwise Fisher's exact test was used. Either the Freeman-Halton extension of Fisher's exact probability test or the chi-squared test with Yates correction was used for tables larger than $2 \times 2$. In the case of significant differences, pair-wise comparisons were carried out by means of Pearson's chi-squared test (if all expected numbers of counts are at least 1); otherwise Fisher's exact test was used. Noncategorical data (e.g., age at seizure onset) were first analyzed by rank-based ANOVA. Two-by-two comparisons were performed by means of either the Man$\mathrm{n}$-Whitney test or $t$ test depending on scale and distribution type. In a comparative analysis of different groups, only those with sample size greater than five were included.

\section{Ethical framework}

All study participants and parents of underage subjects were asked to give their written informed consent after a full explanation of the purposes of the study, MRI/fMRI procedures, SCID I and II interviews and neuropsychological test- ing. The study was approved by the Ethics Committees of the city of Salzburg.

\section{Results}

\section{Plausibility study}

We tested seven patients with JME (four women; mean age $20 \pm 10.8$ years) and seven healthy probands (four women; mean age $25 \pm 4$ years) matched for gender, age, and educational level. The study participants underwent thorough neuropsychological testing, SCID I and II interviews, brain MRI and fMRI with "dynamic fearful faces" and ToM paradigms. Already in this small sample there were obvious deficits in the SCID I and II (in $43 \%$ of patients vs. $0 \%$ in healthy controls). The ToM tests revealed statistically significant more frequent wrong responses in the patient group compared with healthy controls (Faux Pas Test-16\% vs. 6\%; Moving Triangles test $-69 \%$ vs. $53 \%$; Reading the Mind in the Eyes Test- $42 \%$ vs. $28 \%$ ). In patients, there were also clear tendencies of deficits in executive functions and emotion recognition. The structural analysis demonstrated changes in volume and cortical morphology in the left hippocampus, insular and cingulate cortices, as well as frontal lobes in the JME patients compared with healthy controls. The fMRI ToM paradigm also showed aberrant activations in the limbic system in JME patients compared with controls (- Fig. 1). These differences in fMRI analysis, however, were not statistically significant in this small sample.

\section{Discussion}

Here, we present the preliminary results of our study related to emotion recognition and social cognition in patients with JME. Our results, despite a small sample, show clear deficits in patients compared with healthy subjects in executive functions, emotion recognition, and social cognition in both neuropsychological testing and fMRI. This study is ongoing and more data are necessary for further convincing results and sound conclusions. 
Hier steht eine Anzeige.

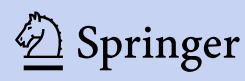


The ability to recognize emotions is the most important social skill. Basic emotions like happiness, fear, disgust, anger, and sadness appear to be cross-cultural with the amygdala playing a crucial role in the processing of cognitive, autonomic, and behavioral responses to emotional stimuli [18]. Social cognition refers to the ability to interpret and predict others' behavior with regard to their beliefs, intentions, feelings, attitudes, and perspectives. Social cognition enables us to interact in complex social environments and to engage in activities that we value most, such as family, friendship, love, and cooperation. Accordingly, impairments in social cognition can have a devastating impact on social interactions, interpersonal relationships, employment, and experiential activities, which are identified as key factors for subjective wellbeing and happiness [19]. The role of the mesial temporal region in decoding emotions has been shown by a number of lesional and functional imaging studies [20]. Involvement of the fronto-limbic structures in both seizure generation in temporal lobe epilepsy (TLE) and recognition of emotions has stimulated numerous studies on social-cognitive abilities in patients with TLE $[21,22]$. The patients with mesial TLE, as compared with patients with epilepsy not originating in the mesial-temporal or frontal lobes and healthy controls, are impaired in their ability to recognize a faux pas-a typical skill [22]. Decision-making also belongs to the various cognitive abilities that fall within the realm of social cognition. Patients with mesial TLE have difficulties learning from feedback and making decisions under uncertainty and ambiguity, as shown by our group [23]. Social-cognition and decision-making abilities of patients with mesial TLE were compared with those with extra-mesial TLE and healthy controls. Patients with mesial TLE were significantly impaired compared with healthy controls on most measures of social cognition. Emotion recognition was more impaired in patients with mesial TLE than in those with extra-mesial TLE [24].

Psychosocial outcome in JME patients is controversial and there are only few studies exploring this domain [25, 26].

Z. Epileptol. 2019·32:177-182 https://doi.org/10.1007/s10309-019-0261-y

(c) The Author(s) 2019

G. Kuchukhidze · J. Höfler · M. Kronbichler · E. Schmid · M. Kirschner - L. Rainer · L. Kronbichler · J. Gaggl · E. Trinka

\section{Emotion recognition and social cognition in juvenile myoclonic epilepsy}

\section{Abstract}

Juvenile myoclonic epilepsy (JME) is the most common idiopathic generalized epilepsy. Disease onset is typically in puberty and poor social adjustment and behavioral disturbances, which resemble frontal lobe dysfunction, are often observed. In recent advanced brain imaging studies on JME patients, emotional and behavioral problems have been associated to subtle structural and functional alterations mainly in frontal cortex and thalamus. There is emerging evidence that patients with abnormal emotion processing and regulation, such as those with bipolar disorder, show disrupted connectivity between limbic structures and frontal cortices. There are no neuroimaging or neuropsychological studies related to emotion processing in patients with JME with a focus on limbic structures. We aimed to address the problem of emotional disturbances and social adjustment in JME patients from multiple aspects through thorough functional and structural assessment, which would potentially enable elaboration of a unifying concept explaining neurobiological background of disturbances in emotional processing and social adjustment in JME patients. Results of this study may potentially enable the development of psychological and pharmacological interventional strategies for managing behavioral disturbances in patients with JME.

Keywords

Theory of Mind $\cdot f M R I \cdot$ Executive functions . Limbic system · Amygdala

\section{Emotionserkennung und soziale Kognition bei juveniler myoklonischer Epilepsie}

\section{Zusammenfassung}

Juvenile myoklonische Epilepsie (JME) ist die am häufigsten auftretende idiopathische generalisierte Epilepsie. Die Erkrankung beginnt typischerweise in der Pubertät und ist häufig mit sozialen Anpassungsstörungen und Verhaltensauffälligkeiten, frontalen Dysfunktionen gleichend, assoziiert. Aktuelle bildgebende Studien bei Patienten mit JME beschreiben eine Assoziation zwischen Beeinträchtigungen von Emotionen und Verhalten mit strukturellen und funktionellen Veränderungen im frontalen Kortex und Thalamus. Bei Patienten mit Beeinträchtigung der Emotionsverarbeitung bzw. -regulation (wie z. B. bei bipolarer Erkrankung) wurden außerdem Störungen zwischen dem Regelkreis des limbischen Systems und dem frontalen Kortex beschrieben. Bis dato gibt es bei Patienten mit JME keine bildgebenden und neuropsychologischen Untersuchungen hinsichtlich des limbischen Systems und der Emotionsverarbeitung. Ziel der Studie ist es daher, dies unter verschiedenen Aspekten mittels funktioneller und struktureller Methoden darzustellen und ein einheitliches, auf neurobiologischen Erkenntnissen basierendes Konzept zu etablieren. Die Ergebnisse dieser Studie können wesentlich zur Entwicklung von psychologischen und pharmakologischen Strategien bei Patienten mit JME beitragen und damit zu einem besseren Management der sozialen Anpassungs- und Verhaltensstörungen führen.

\section{Schlüsselwörter}

Theory of Mind · Funktionelle Magnetresonanztomographie - Exekutive Funktionen . Limbisches System · Amygdala
Poor seizure control and lifelong treatment with antiepileptic drugs were associated with difficulties in social adjustment and occupational integration [26]. In a study comparing long-term social outcome in JME and absence epilepsy patients by means of a structured ques- tionnaire, a satisfying outcome was observed in JME [25]. These results, however, could have been influenced by the benign course of epilepsy in the majority of patients who were on monotherapy of antiepileptic drugs, had long and stable physician-patient relationship, and 

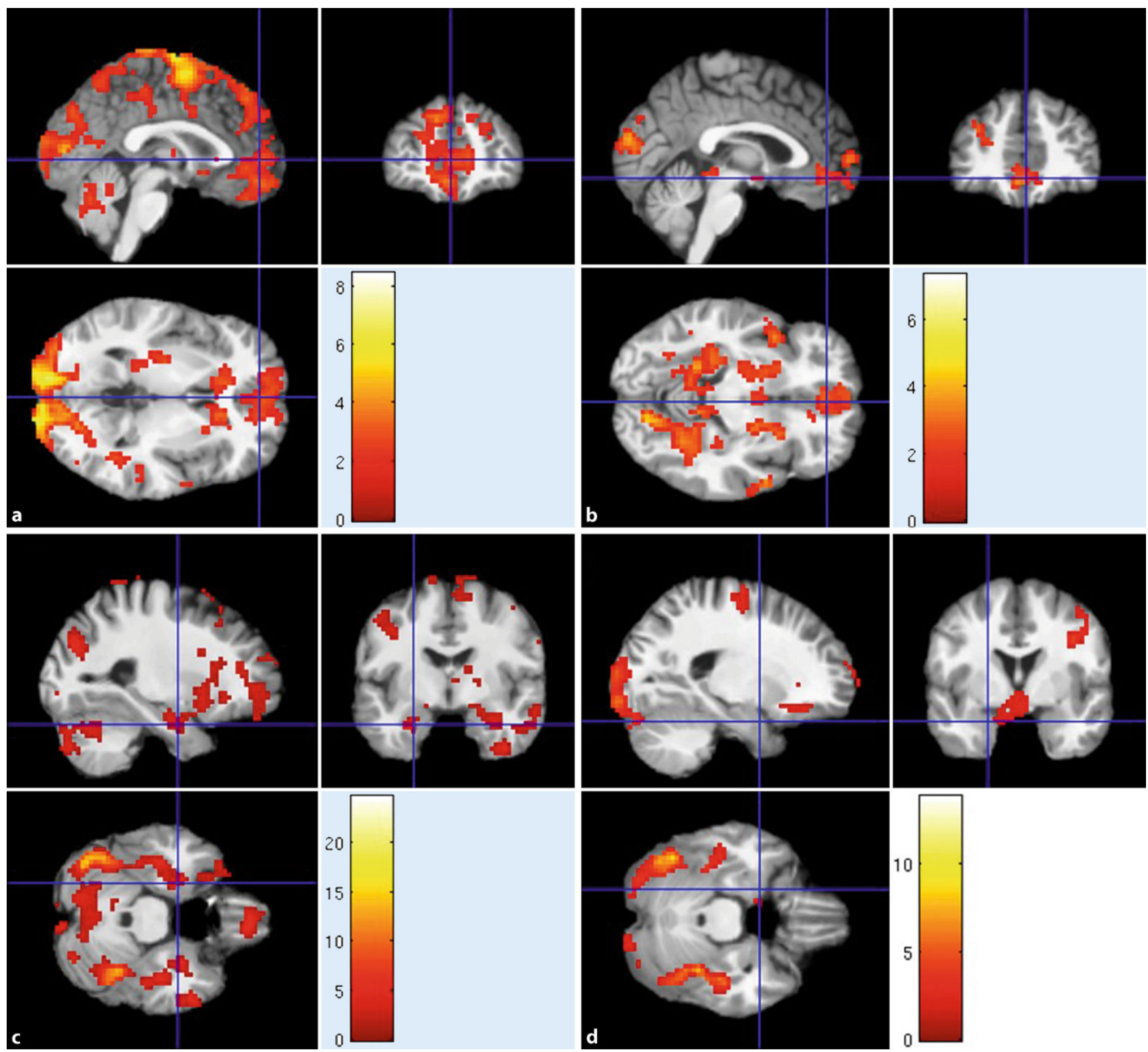

Fig. $1 \Delta$ Results of the theory of mind (ToM; first row) and Emotion (second row) fMRI paradigms in healthy controls (a, c) and patients with juvenile myoclonic epilepsy (JME; b, d):ToM paradigm: Larger BOLD cluster in anterior cingulate bilaterally in healthy control subject (a) compared with JME patient (b). Emotion paradigm: BOLD clusters in amygdala bilaterally in healthy control subject (c) vs. absent activation in JME patient (d)

were older. This study did not investigate the influence of psychiatric comorbidity on social outcome, which was reported previously [8]. Early recognition of personality traits and effective medical and social interventions may prevent harmful consequences of poor psychosocial adjustment [27]. Indeed, behavioral treatment for adolescents and young adult patients with difficult-to-treat JME was associated with improved seizure control and reduction of anxiety and insomnia [28].
Imaging studies of JME patients have focused mainly on thalamo-cortical connections and most have assessed morphological aspects (e.g., cortical thickness, connections between given brain structures) in isolation without their functional correlates and potential psychosocial consequences [29]. We intend to overcome these limitations by utilizing a novel imaging approach and looking for possible morphological and functional alterations from different perspectives. Another serious shortcoming of previous JME imaging studies is the almost exclusive focus on adult populations of JME patients with many years of seizure disorder behind them [29]. Juvenile myoclonic epilepsy manifests itself in early adolescence with a peak at around 14-16 years and therefore it is of decisive importance to carry out imaging studies on young patients with new-onset JME.

In our upcoming study, we plan to include prospectively at least 50 patients with newly manifested JME (within the 
first 3 years of disease onset). We aim to compare imaging and neuropsychological data of younger patients with newly manifested JME with those who have longstanding JME in order to determine whether cerebral structural/functional changes are intrinsic to the epileptic condition or whether they represent the result of longstanding seizure activity. Cognitive dysfunction and increased cortical excitability have been reported in siblings of JME patients [30]. We aim to further investigate the cognitive profile and imaging correlates in the siblings of JME patients with a focus on emotion recognition and social cognition.

Other important questions concerning the impact of various disease variables (seizure types, frequency, seizure freedom, EEG patterns, etc.) as well as the effect of the anti-epileptic drug treatment on emotion recognition and social cognition will be addressed in our upcoming study.

\section{Corresponding address}

\section{G. Kuchukhidze, MD, PhD}

Department of Neurology, Christian Doppler Klinik, Paracelsus Medical University of Salzburg

Ignaz Harrer Straße 79, 5020 Salzburg, Austria g.kuchukhidze@salk.at

Funding. This study has been supported by FWF (Fonds zur Förderung der wissenschaftlichen Forschung), Austrian Science Fund; Project number KLI 543 B-27.

Funding. Open access funding provided by Paracelsus Medical University.

\section{Compliance with ethical guidelines}

Conflict of interest G. Kuchukhidze, J. Höfler, M. Kronbichler, E. Schmid, M. Kirschner, L. Rainer, L. Kronbichler, J. Gaggl, and E. Trinka declare that the research was conducted in the absence of any commercial or financial relationships that could be construed as a potential conflict of interest.

All procedures followed were in accordance with the ethical standards of the responsible committee on human experimentation (institutional and national) and with the Helsinki Declaration of 1975 (in its most recently amended version). Informed consent was obtained from all patients included in the study. The study has been conducted in accordance to the WMA Declaration of Helsinki and Good Clinical Practice (GCP) Guidelines.
Open Access. This article is distributed under the terms of the Creative Commons Attribution 4.0 International License (http://creativecommons.org/licenses/by/ 4.0/), which permits unrestricted use, distribution, and reproduction in any medium, provided you give appropriate credit to the original author(s) and the source, provide a link to the Creative Commons license, and indicate if changes were made.

\section{References}

1. Zifkin B, Andermann E, Andermann F (2005) Mechanisms, genetics, and pathogenesis of juvenile myoclonic epilepsy. Curr Opin Neurol 18:147-153

2. Janz D (1985) Epilepsy with Impulsive Petit Mal (Juvenile Myoclonic Epilepsy). Acta Neurol Scand 72:449-459

3. Montalenti E, Imperiale D, Rovera A, Bergamasco B, Benna $P$ (2001) Clinical features, EEG findings and diagnostic pitfalls in juvenile myoclonic epilepsy: a series of 63 patients. J Neurol Sci 184:65-70

4. Koepp MJ, Woermann F, Savic I, Wandschneider B (2013) Juvenile myoclonic epilepsy-neuroimaging findings. Epilepsy Behav 28(Suppl 1):S40-S44

5. Kim JH, Kim JH, Suh SI, Seol HY, Jung KY, Koh SB (2009) Thalamofrontal circuitry abnormalities and frontal executive dysfunction in juvenile myoclonic epilepsy: diffusion tensor Mri and neuropsychological studies. Epilepsia 50:81

6. Wandschneider B, Centeno M, Vollmar C et al (2013) Risk-taking behavior in juvenile myoclonic epilepsy. Epilepsia 54:2158-2165

7. Zamarian L, Hofler J, Kuchukhidze G et al (2013) Decision making in juvenile myoclonic epilepsy. JNeurol 260:839-846

8. Trinka E, Kienpointner G, Unterberger l et al (2006) Psychiatric comorbidity in juvenile myoclonic epilepsy. Epilepsia 47:2086-2091

9. Ladouceur CD, Farchione T, Diwadkar V et al (2011) Differential patterns of abnormal activity and Connectivity in the amygdala-prefrontal circuitry in bipolar-I and bipolar-NOS youth. J Am Acad Child Adolesc Psychiatry 50:1275-1289

10. Versace A, Thompson WK, Zhou DL et al (2010) Abnormal left and right amygdala-orbitofrontal cortical functional connectivity to emotional faces: state versus trait vulnerability markers of depression in bipolar disorder. Biol Psychiatry 67:422-431

11. First MB, Spitzer RL, Gibbon M, Williams JBW (1995) The structured clinical interview for Dsmiii-R personality-disorders (Scid-ii) .1. description. JPers Disord 9:83-91

12. First MB, Spitzer RL, Gibbon $M$ et al (1995) The structured clinical interview for Dsm-iii-R personality-disorders (Scid-ii) .2. Multisite testretest reliability study. J Pers Disord 9:92-104

13. Thomas LA, De Bellis MD, Graham R, Labar KS (2007) Development of emotional facial recognition in late childhood and adolescence. DevSci 10:547-558

14. Baron-CohenS, WheelwrightS(2004) The empathy quotient: an investigation of adults with Asperger syndrome or high functioning autism, and normal sex differences. J Autism Dev Disord 34:163-175

15. Abell F, Happe F, Frith U (2000) Do triangles play tricks? Attribution of mental states to animated shapes in normal and abnormal development. Cogn Dev 15:1-16

16. Baron-Cohen S, Wheelwright S, Hill J, Raste $Y$, Plumb I (2001) The "Reading the Mind in the Eyes"
Test revised version: a study with normal adults, and adults with Asperger syndrome or highfunctioning autism. J Child Psychol Psychiatry 42:241-251

17. Stone VE, Baron-Cohen S, Knight RT (1998) Frontal lobe contributions to theory of mind. J Cogn Neurosci 10:640-656

18. Adolphs R (2002) Neural systems for recognizing emotion. Curr Opin Neurobiol 12:169-177

19. Helliwell JF, Putnam RD (2004) The social context of well-being. Philos Trans R Soc Lond, B, Biol Sci 359:1435-1446

20. Adolphs R (2008) Fear, faces, and the human amygdala. Curr Opin Neurobiol 18:166-172

21. Meletti S, Benuzzi F, Cantalupo G, Rubboli G, Tassinari CA, Nichelli P (2009) Facial emotion recognition impairment in chronic temporal lobe epilepsy. Epilepsia 50:1547-1559

22. Schacher M, Winkler R, Grunwald T et al (2006) Mesial temporal lobe epilepsy impairs advanced social cognition. Epilepsia 47:2141-2146

23. Delazer M, Zamarian L, Bonatti E et al (2011) Decision making under ambiguity in temporal lobe epilepsy: does the location of the underlying structural abnormality matter? Epilepsy Behav 20:34-37

24. Broicher SD, Kuchukhidze G, Grunwald T, Kramer G, Kurthen M, Jokeit H (2012) "Tell me how do I feel"-Emotion recognition and theory of mind in symptomatic mesial temporal lobe epilepsy. Neuropsychologia 50:118-128

25. Holtkamp $M$, Senf $P$, Kirschbaum $A$, Janz D (2014) Psychosocial long-term outcome in juvenile myoclonic epilepsy. Epilepsia 55:1732-1738

26. Schneider-von Podewils F, Gasse C, Geithner J et al (2014) Clinical predictors of the long-term social outcome and quality of life in juvenile myoclonic epilepsy: 20-65years of follow-up. Epilepsia 55:322-330

27. Lund M, Reintoft $H$, Simonsen (1976) Controlled sociological and psychological-study of patients with juvenile myoclonus epilepsy. Nervenarzt 47:708-712

28. Martinovic Z (2001) Adjunctive behavioural treatment in adolescents and young adults with juvenile myoclonic epilepsy. Seizure Eur JEpilepsy 10:42-47

29. Vollmar C, O'Muircheartaigh J, Symms MR et al (2012) Altered microstructural connectivity in juvenile myoclonic epilepsy The missing link. Neurology 78:1555-1559

30. Akgun Y, Soysal A, Atakli D, Yuksel B, Dayan C, Arpaci $B$ (2009) Cortical excitability in juvenile myoclonic epileptic patients and their asymptomatic siblings: a transcranial magnetic stimulation study. Seizure Eur JEpilepsy 18:387-391 